\title{
The Essential Guide to HTML5 and CSS3 Web Design
}

\author{
Craig Grannell \\ Victor Sumner \\ Dionysios Synodinos
}

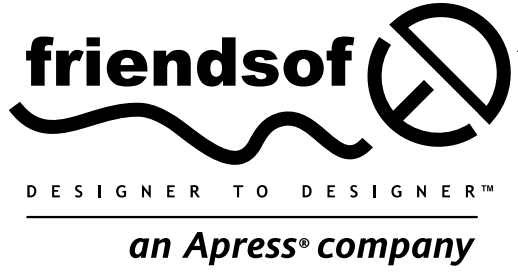




\title{
The Essential Guide to HTML5 and CSS3 Web Design
}

\begin{abstract}
Copyright @ 2012 by Craig Grannell, Victor Sumner, Dionysios Synodinos
This work is subject to copyright. All rights are reserved by the Publisher, whether the whole or part of the material is concerned, specifically the rights of translation, reprinting, reuse of illustrations, recitation, broadcasting, reproduction on microfilms or in any other physical way, and transmission or information storage and retrieval, electronic adaptation, computer software, or by similar or dissimilar methodology now known or hereafter developed. Exempted from this legal reservation are brief excerpts in connection with reviews or scholarly analysis or material supplied specifically for the purpose of being entered and executed on a computer system, for exclusive use by the purchaser of the work. Duplication of this publication or parts thereof is permitted only under the provisions of the Copyright Law of the Publisher's location, in its current version, and permission for use must always be obtained from Springer. Permissions for use may be obtained through RightsLink at the Copyright Clearance Center. Violations are liable to prosecution under the respective Copyright Law.
\end{abstract}

ISBN 978-1-4302-3786-0

ISBN 978-1-4302-3787-7 (eBook)

Trademarked names, logos, and images may appear in this book. Rather than use a trademark symbol with every occurrence of a trademarked name, logos, or image we use the names, logos, or images only in an editorial fashion and to the benefit of the trademark owner, with no intention of infringement of the trademark.

The use in this publication of trade names, service marks, and similar terms, even if they are not identified as such, is not to be taken as an expression of opinion as to whether or not they are subject to proprietary rights.

While the advice and information in this book are believed to be true and accurate at the date of publication, neither the authors nor the editors nor the publisher can accept any legal responsibility for any errors or omissions that may be made. The publisher makes no warranty, express or implied, with respect to the material contained herein.

Distributed to the book trade worldwide by Springer Science+Business Media New York, 233 Spring Street, 6th Floor, New York, NY 10013. Phone 1-800-SPRINGER, fax (201) 348-4505, e-mail orders-ny@springer-sbm.com, or visit WWw. springeronline.com.

For information on translations, please e-mail rights@apress.com or visitwww.apress.com.

Apress and friends of ED books may be purchased in bulk for academic, corporate, or promotional use. eBook versions and licenses are also available for most titles. For more information, reference our Special Bulk Sales-eBook Licensing web page at wWw. apress.com/bulk-sales.

Any source code or other supplementary materials referenced by the author in this text is available to readers at www.apress.com. For detailed information about how to locate your book's source code, go to www. apress.com/source-code.

\section{Credits}

\section{President and Publisher:} Paul Manning

Lead Editor: Tom Welsh

Technical Reviewer: Jeffrey Sambells

Editorial Board:

Steve Anglin, Ewan Buckingham, Gary Cornell, Louise Corrigan, Morgan Ertel, Jonathan Gennick, Jonathan Hassell, Robert Hutchinson, Michelle Lowman, James Markham, Matthew Moodie, Jeff Olson, Jeffrey Pepper, Douglas Pundick, Ben Renow-Clarke, Dominic Shakeshaft, Gwenan Spearing, Matt Wade, Tom Welsh

Coordinating Editors: Jessica Belanger, Anamika Panchoo

\section{Copy Editor:}

Kim Wimpsett

Compositor:

Bytheway Publishing Services

\section{Indexer:}

SPi Global

Artist:

SPi Global

\section{Cover Image Artist:}

Corné van Dooren

\section{Cover Designer:}

Anna Ishchenko 
Dedicated to my grandmother, Ellen, whose passion for life has always inspired me to take on any challenge.

\section{-Victor Sumner}

I dedicate this book to my wonderful family.

To my loving mother, Aggeliki.

To my beautiful wife, Elisa.

To my beloved daughter, Aggeliki.

To my precious newborn son.

You make me feel like the luckiest person alive.

—Dionysios Synodinos 


\section{Contents at a Glance}

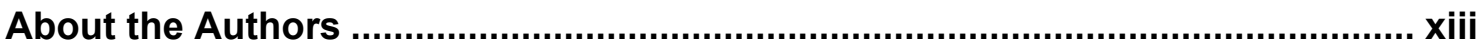

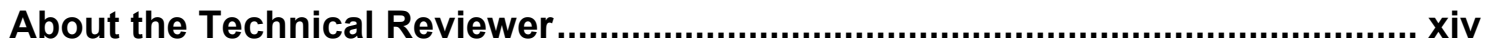

About the the Cover Image Designer ................................................................ XV

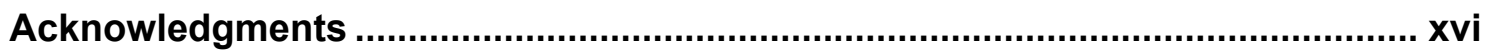

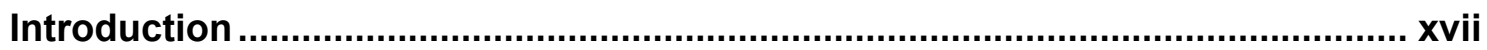

Chapter 1: An Introduction to Web Design ...................................................... 1

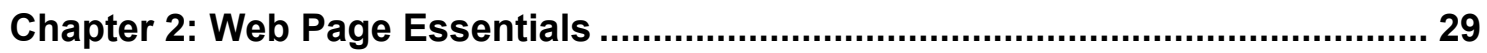

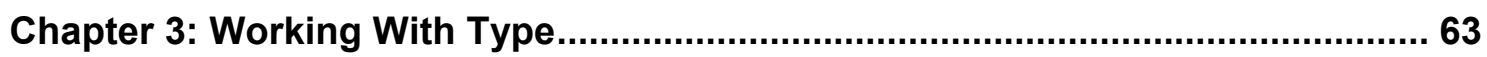

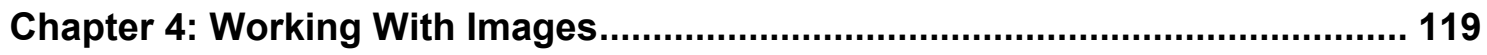

Chapter 5: Using Links and Creating Navigation ................................................ 145

Chapter 6: Tables: How Nature (and the W3C) Intended ...................................... 227

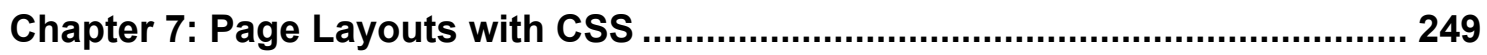

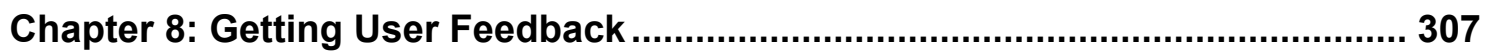

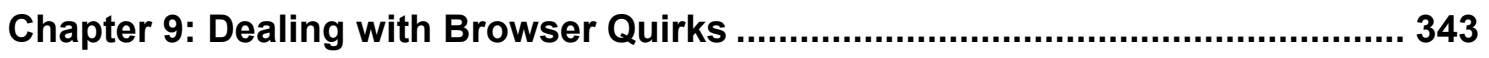

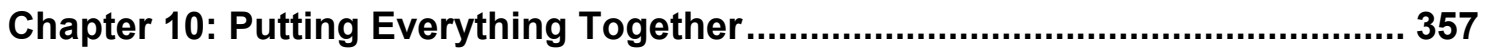

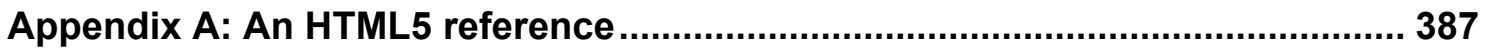

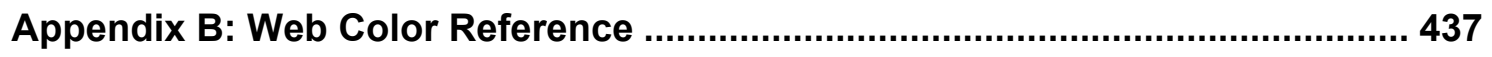

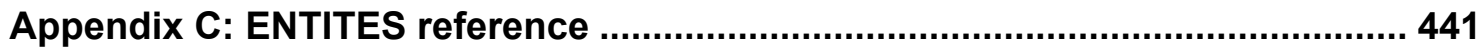

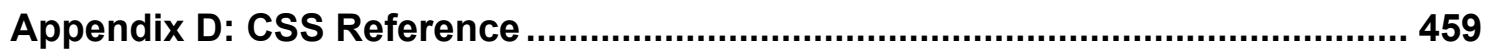

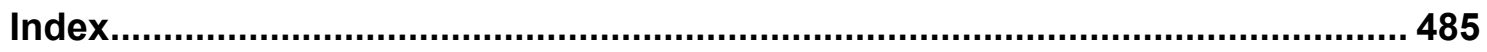




\section{Contents}

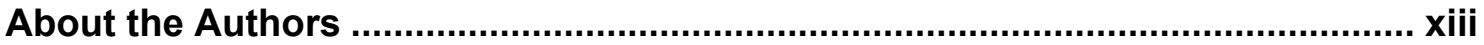

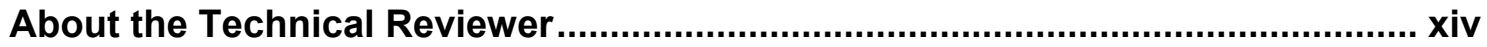

About the the Cover Image Designer ................................................................ XV

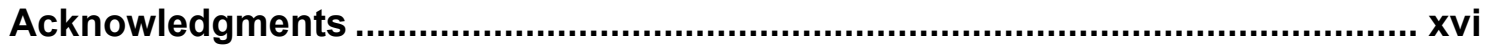

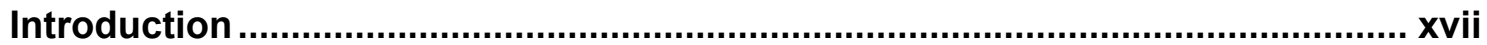

Chapter 1: An Introduction to Web Design .......................................................... 1

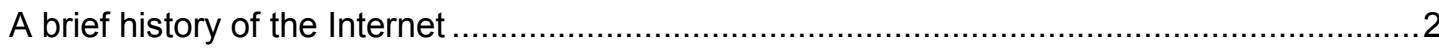

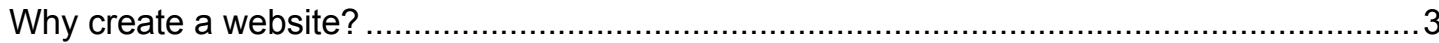

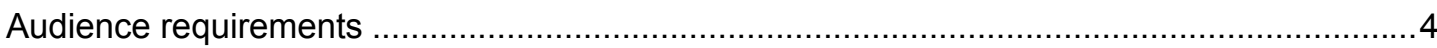

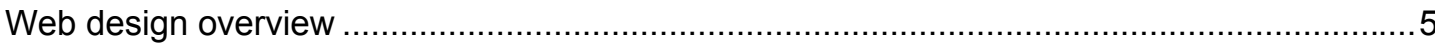

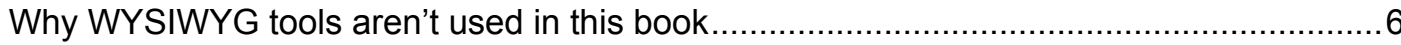

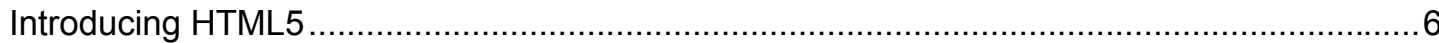

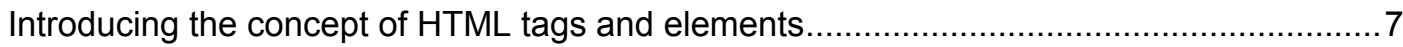

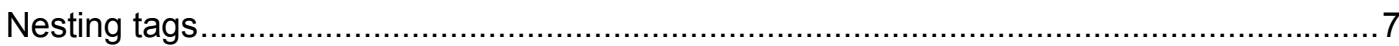

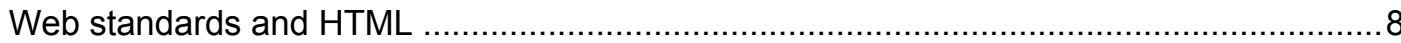

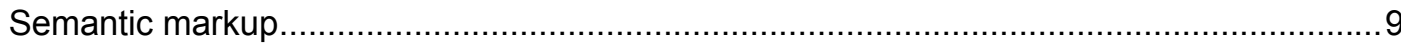

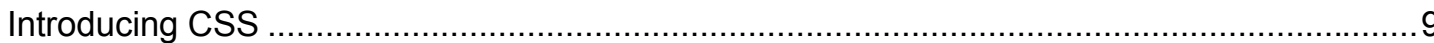

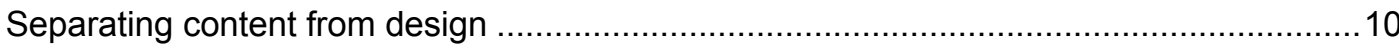

The rules of CSS

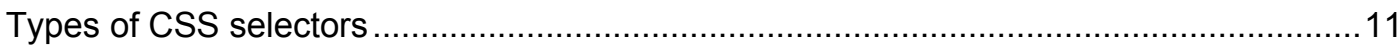

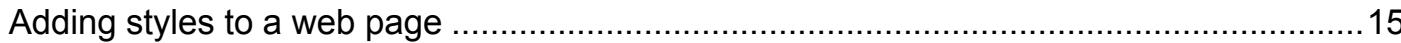

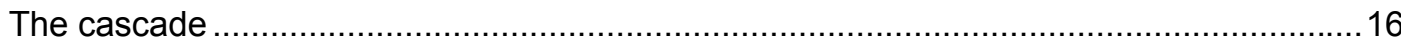

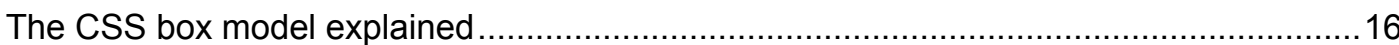

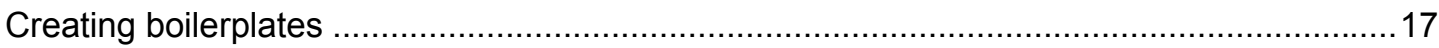

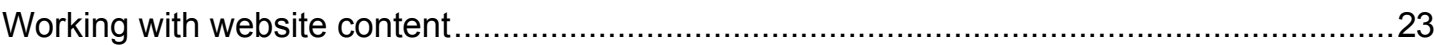

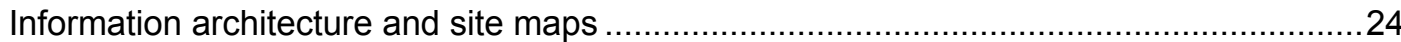

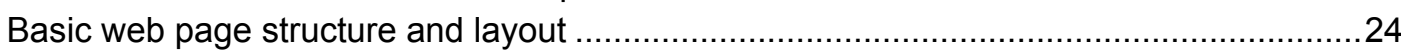

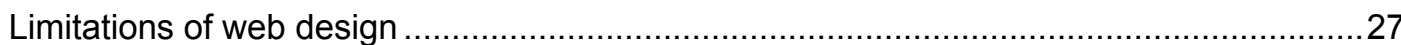

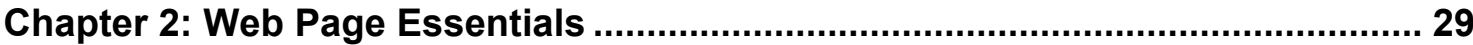

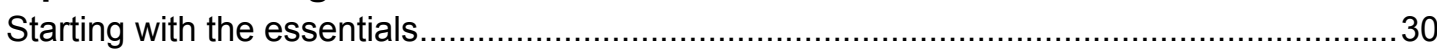

HTML vs. XHTML .

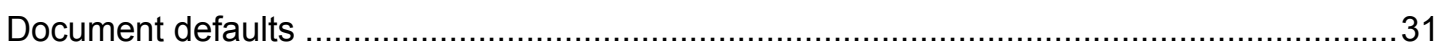




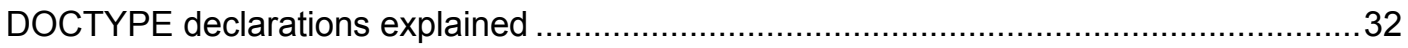

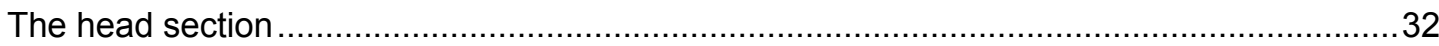

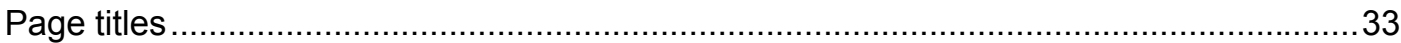

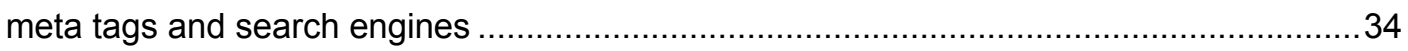

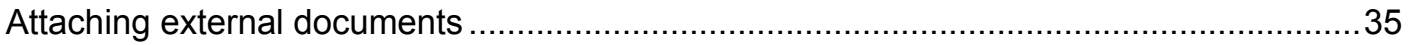

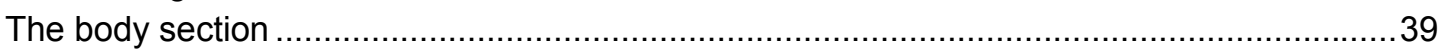

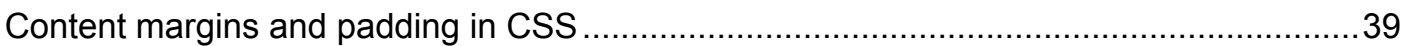

Zeroing margins and padding on all elements ........................................................40

Working with CSS shorthand for boxes..................................................................4 40

Setting a default font and font color .............................................................. 41

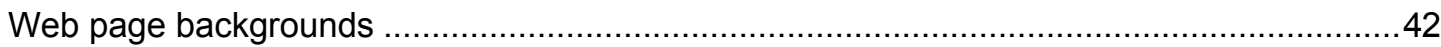

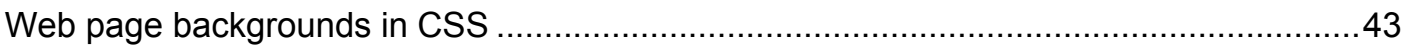

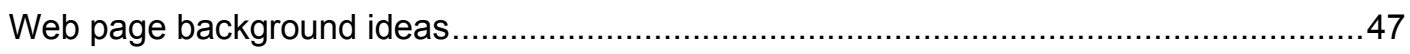

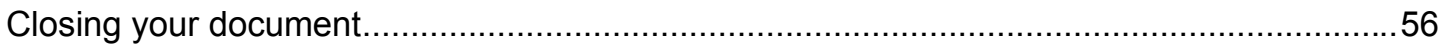

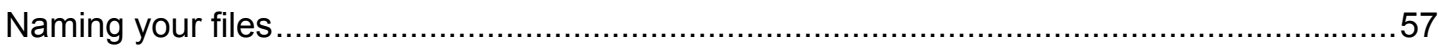

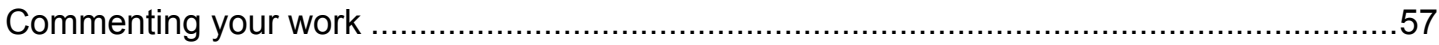

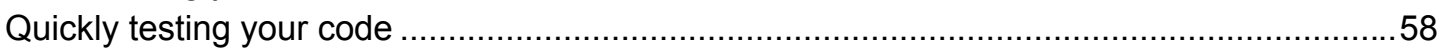

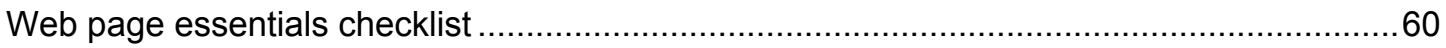

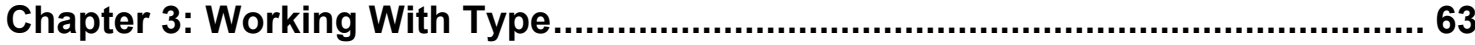

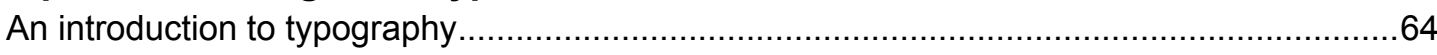

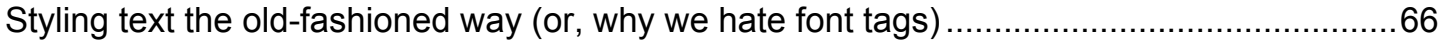

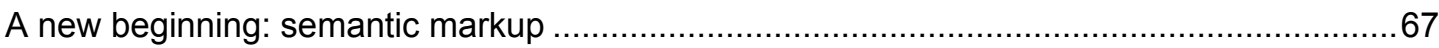

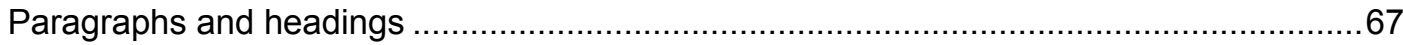

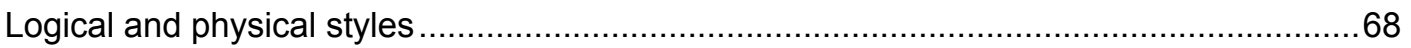

The importance of well-formed markup ............................................................... 71

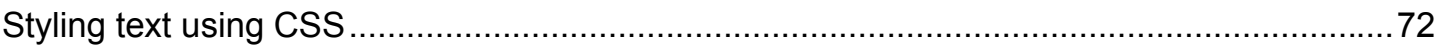

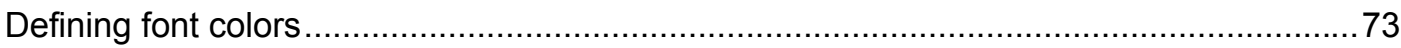

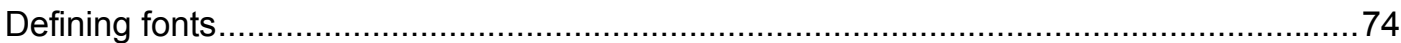

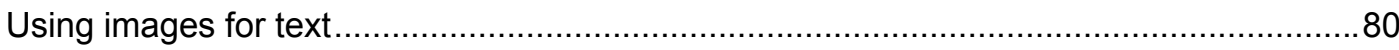

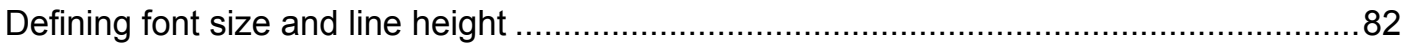

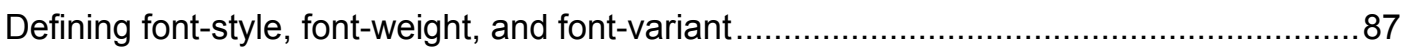

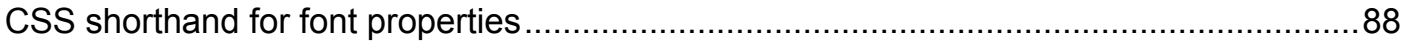

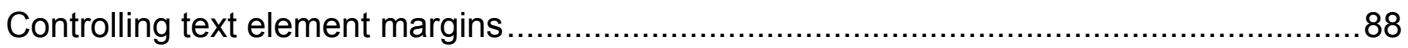

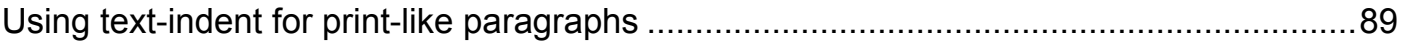

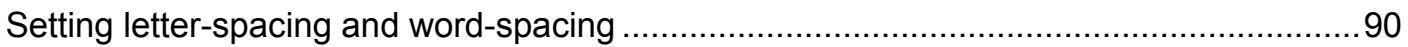

Controlling case with text-transform ................................................................ 91

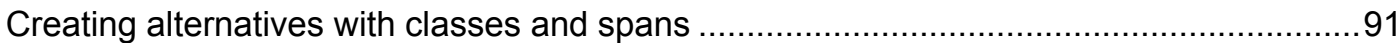




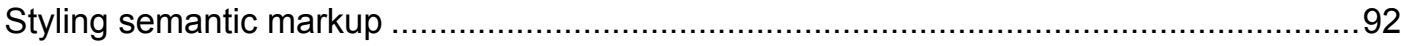

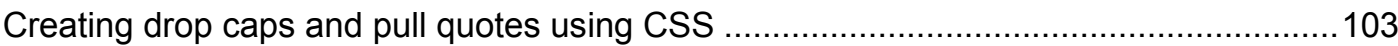

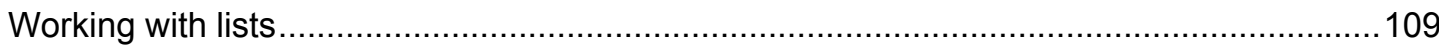

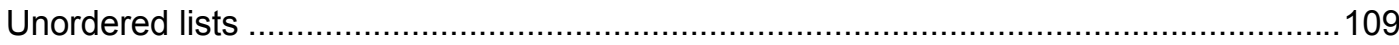

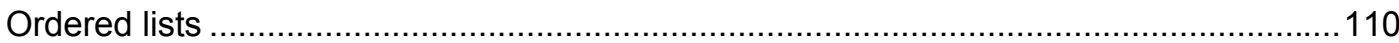

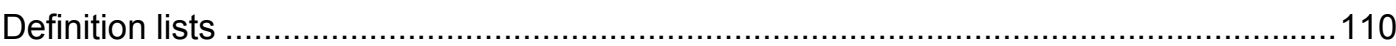

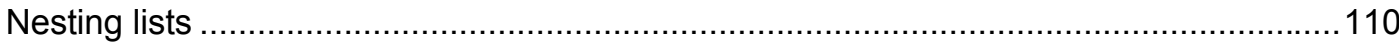

Styling lists with CSS

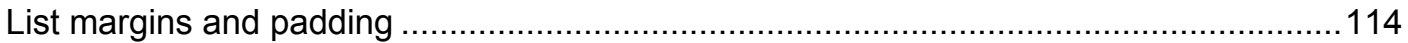

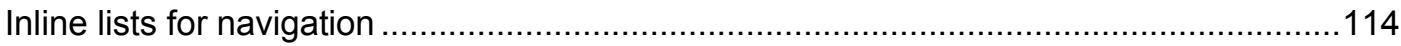

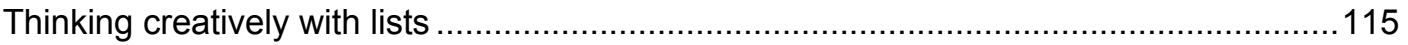

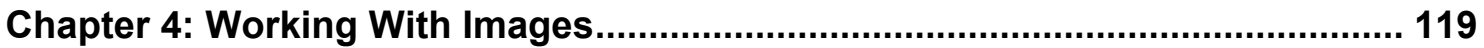

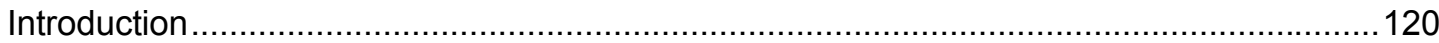

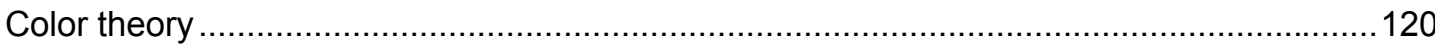

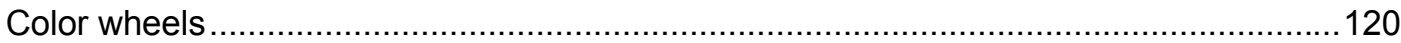

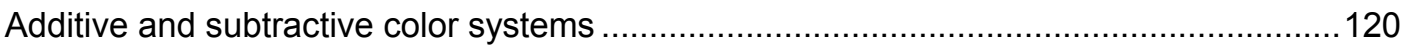

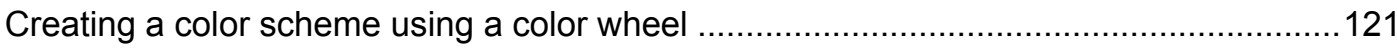

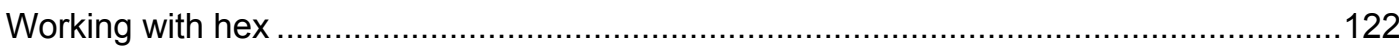

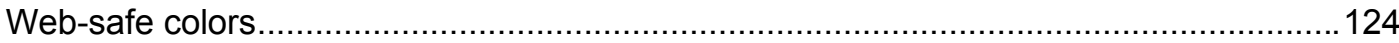

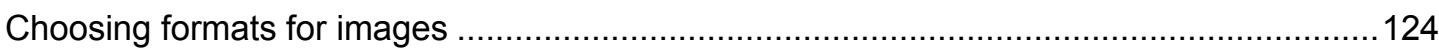

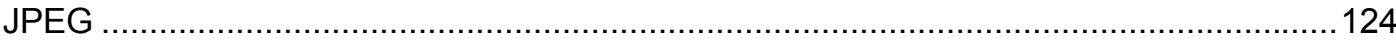

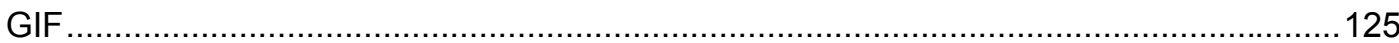

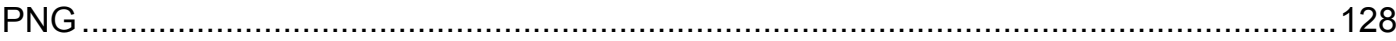

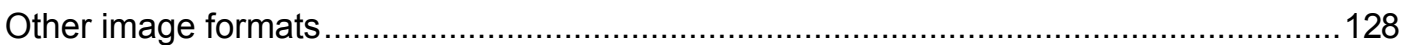

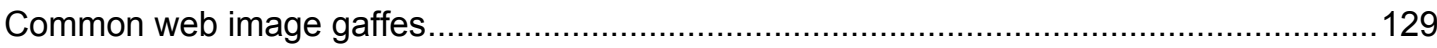

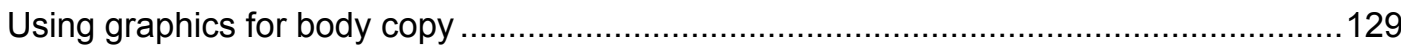

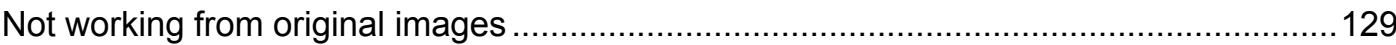

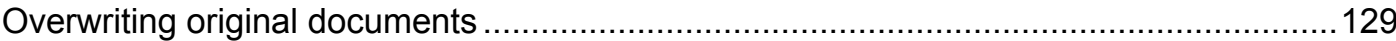

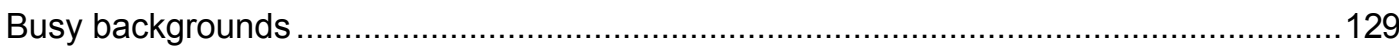

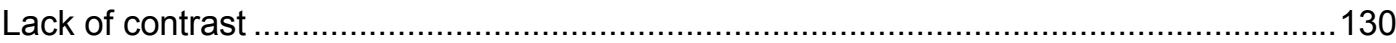

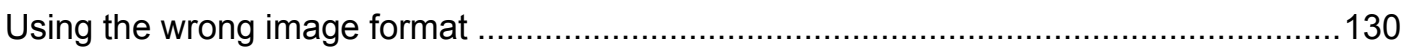

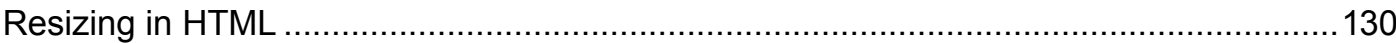

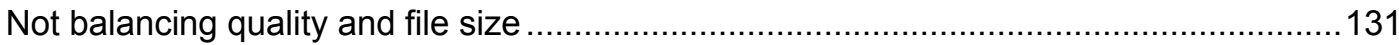

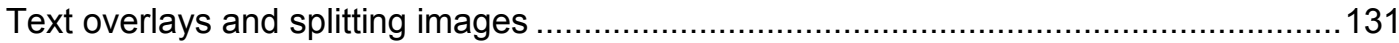

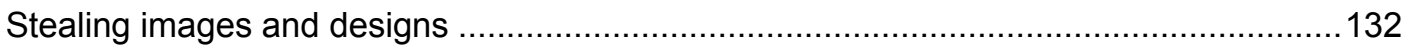

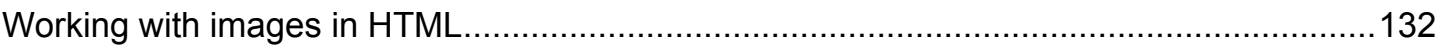

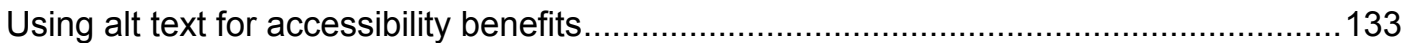


Descriptive alt text for link-based images....................................................... 133

Null alt attributes for interface images ............................................................... 133

Using alt and title text for tooltips .................................................................. 134

Using CSS when working with images............................................................. 134

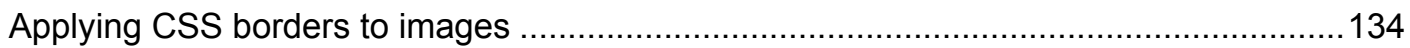

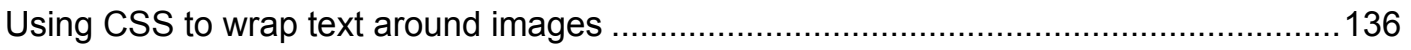

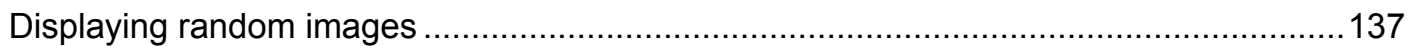

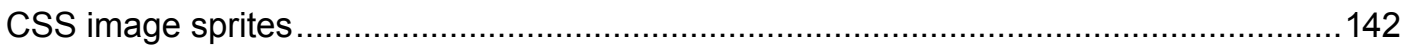

Chapter 5: Using Links and Creating Navigation ........................................ 145

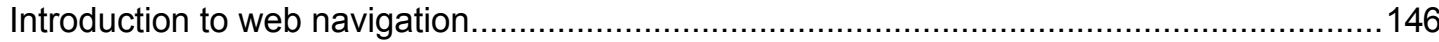

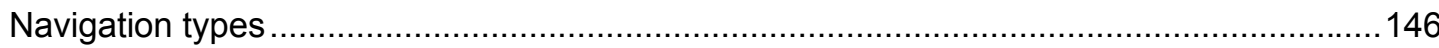

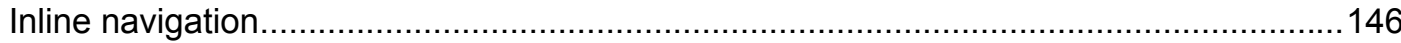

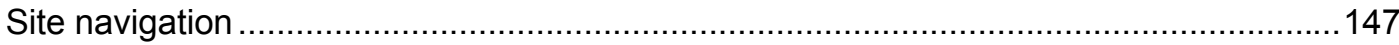

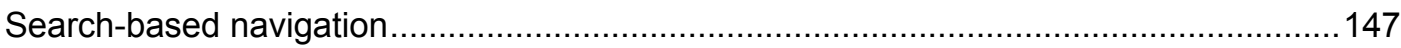

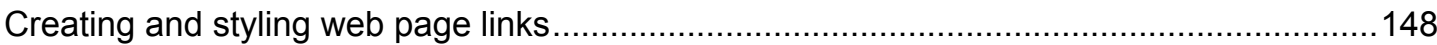

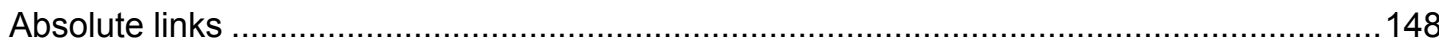

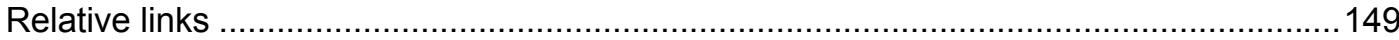

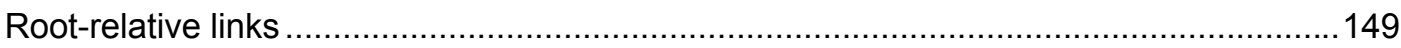

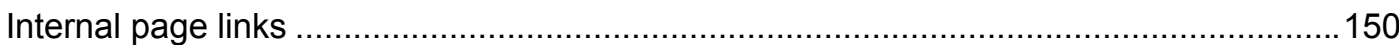

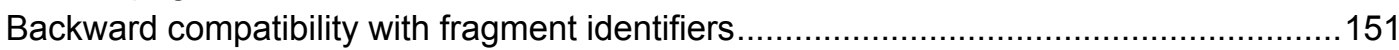

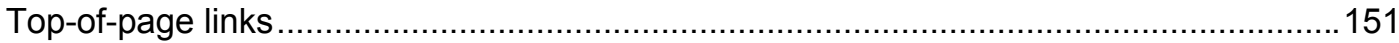

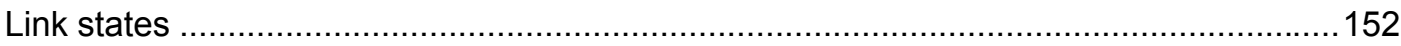

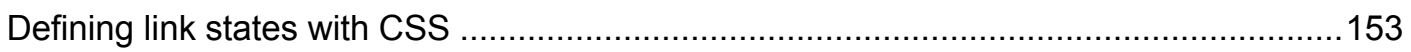

Correctly ordering link states ............................................................................... 153

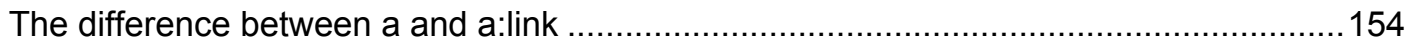

Editing link styles using CSS ........................................................................... 154

Multiple link states: The cascade .................................................................... 156

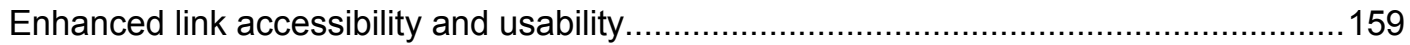

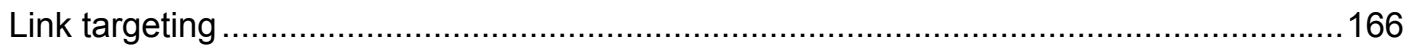

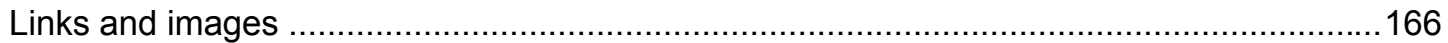

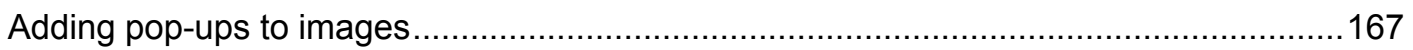

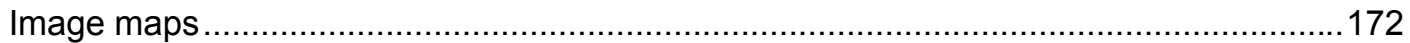

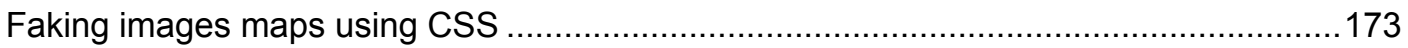

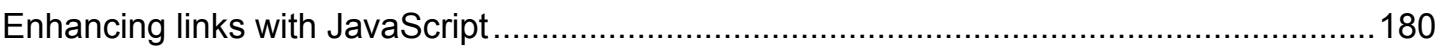

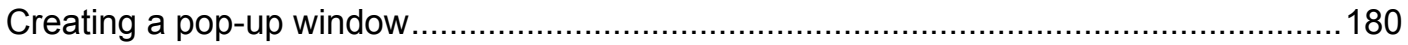

Creating an online gallery ................................................................................ 182

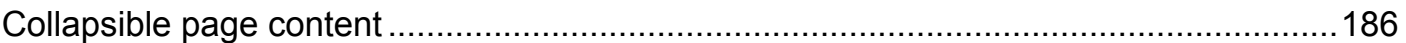




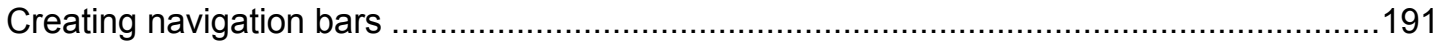

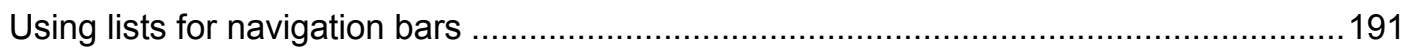

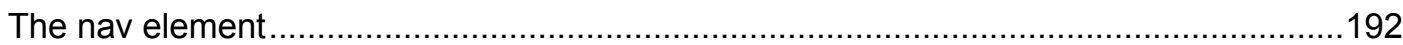

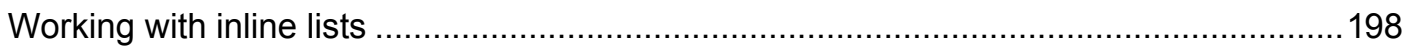

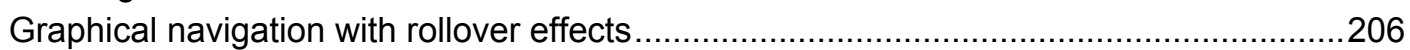

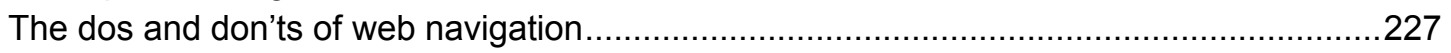

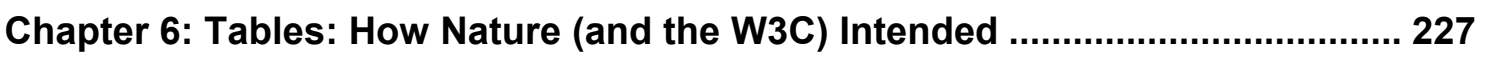

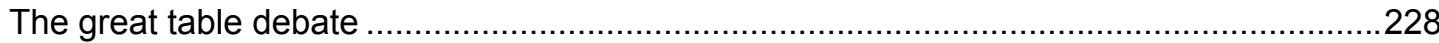

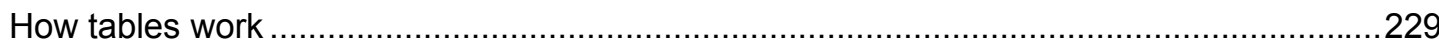

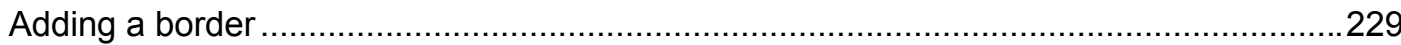

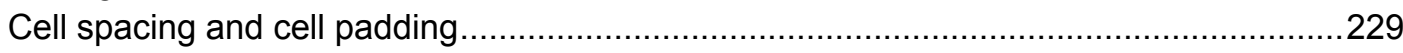

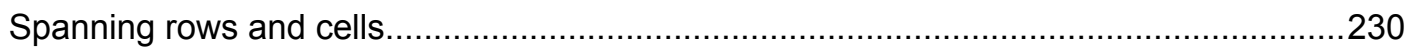

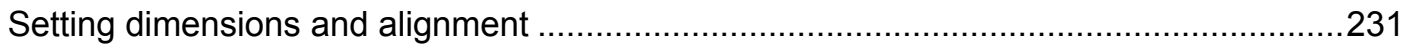

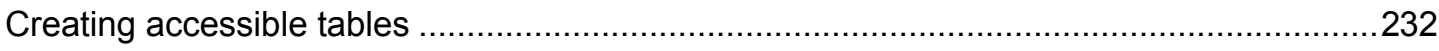

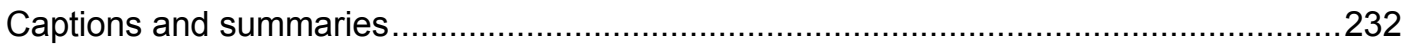

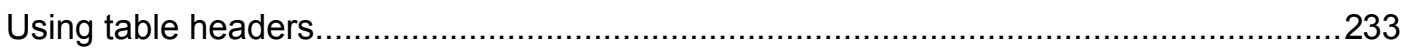

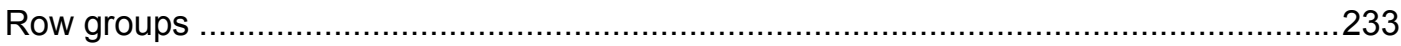

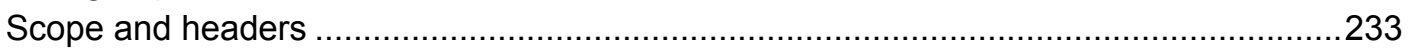

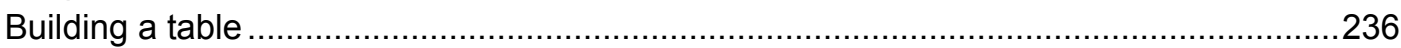

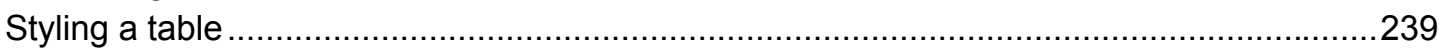

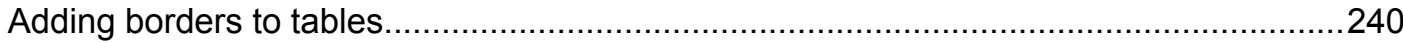

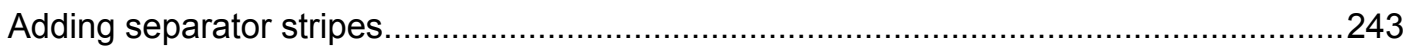

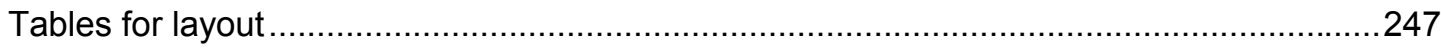

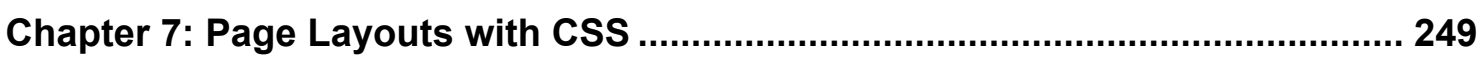

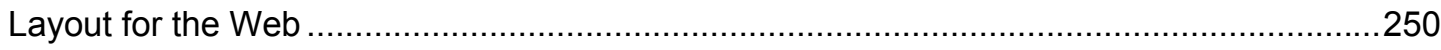

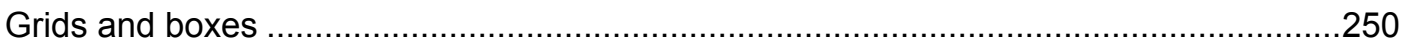

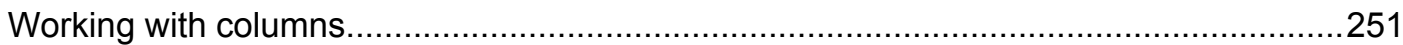

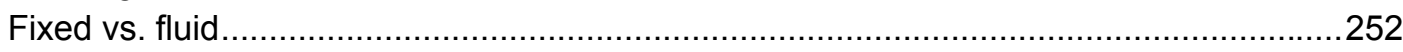

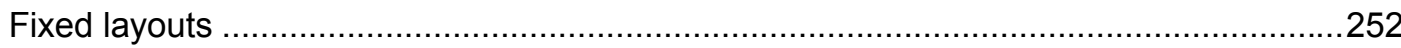

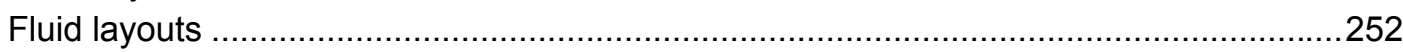

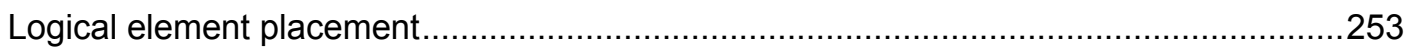

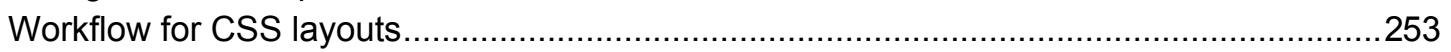

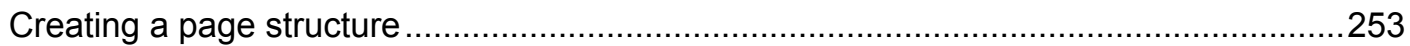

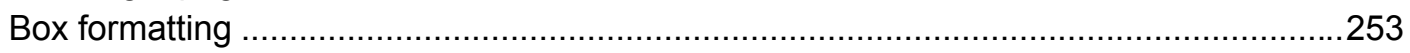

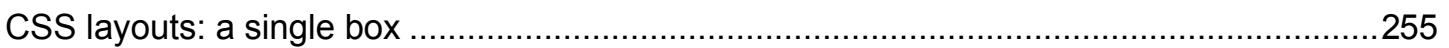

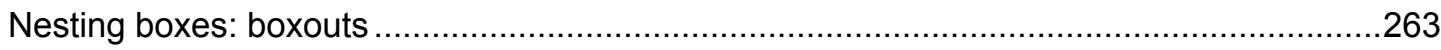

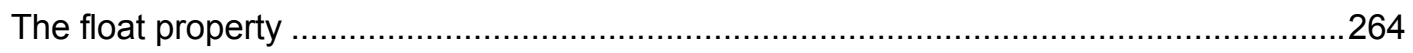


Advanced layouts with multiple boxes and columns .............................................268

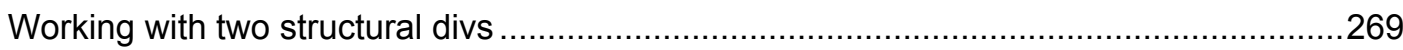

Placing columns within wrappers and clearing floated content.................................280

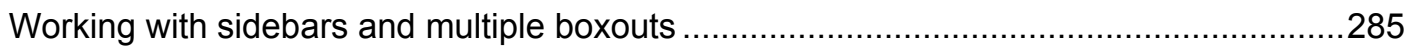

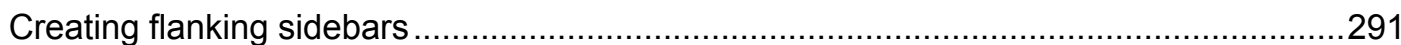

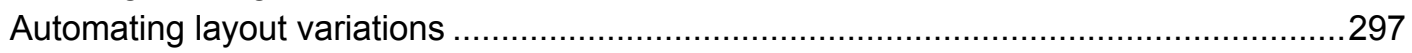

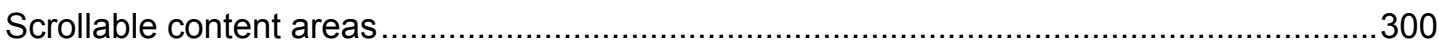

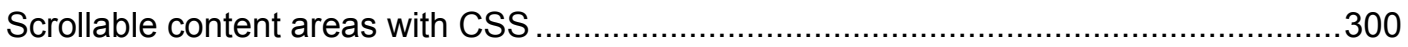

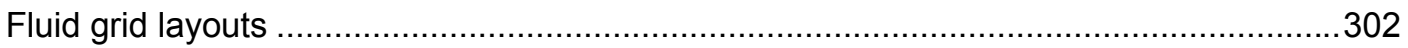

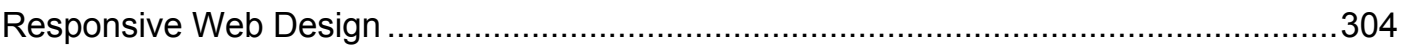

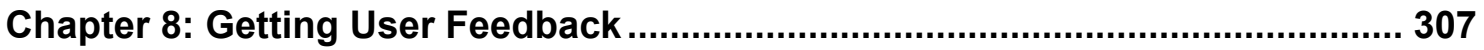

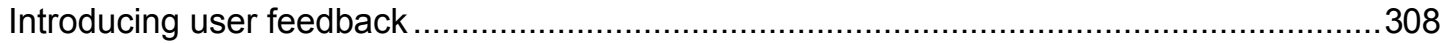

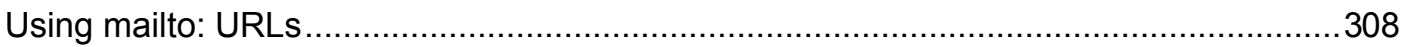

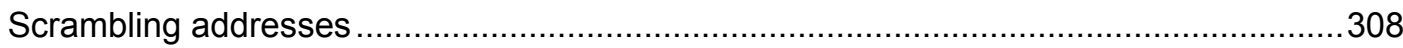

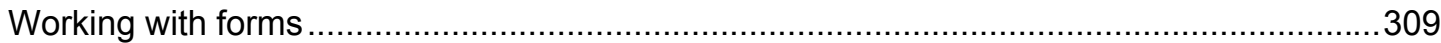

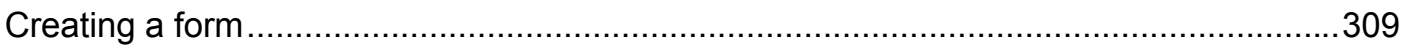

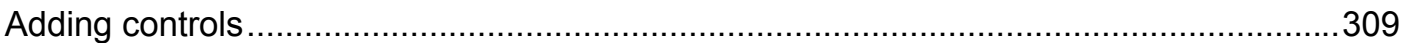

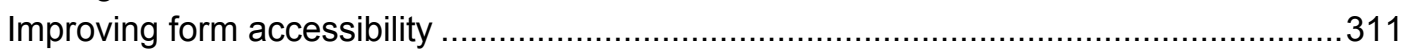

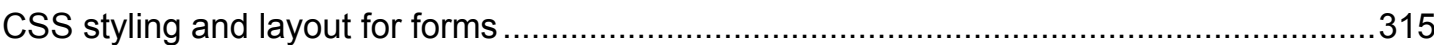

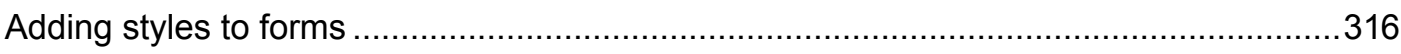

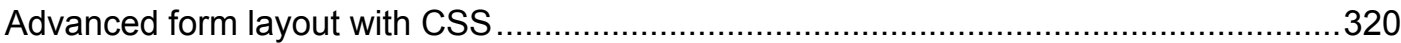

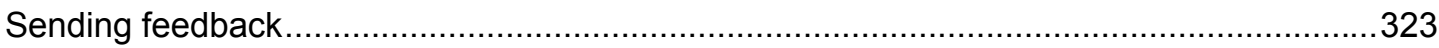

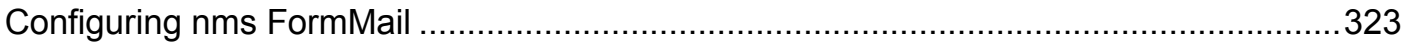

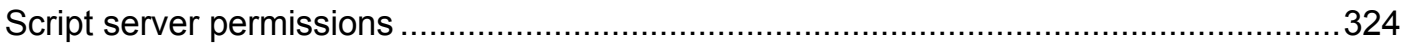

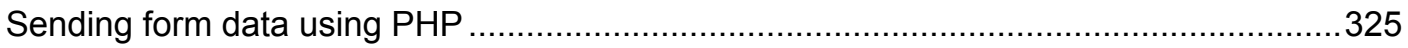

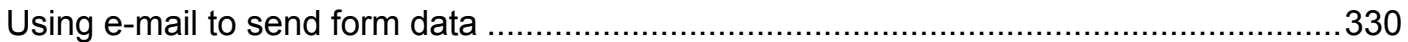

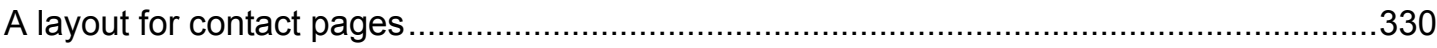

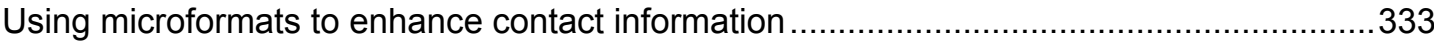

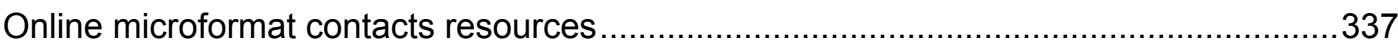

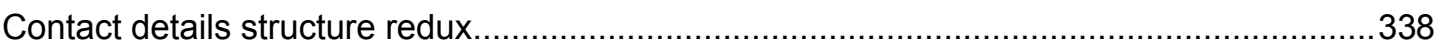

Chapter 9: Dealing with Browser Quirks ….................................................... 343

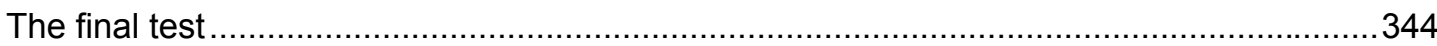

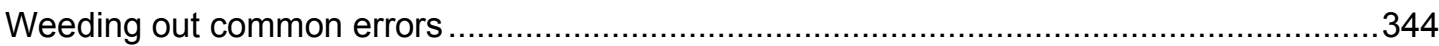

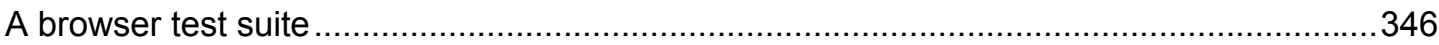

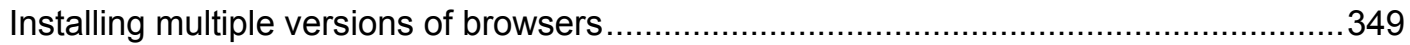

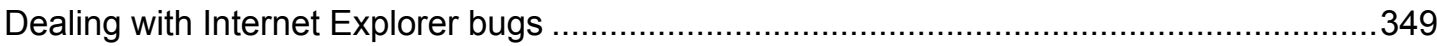

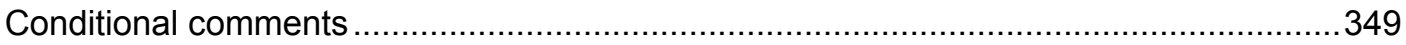




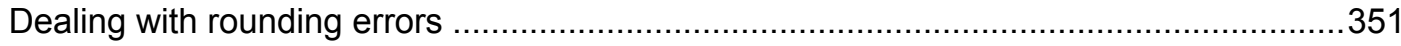

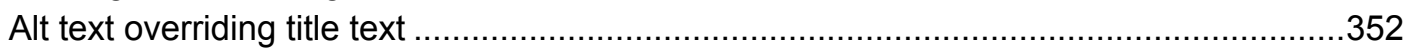

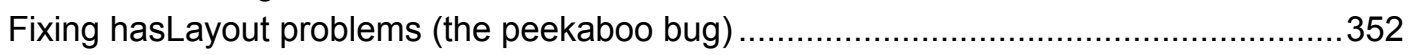

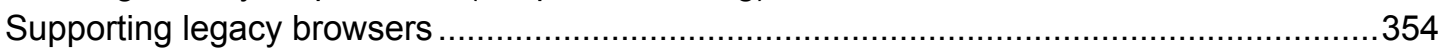

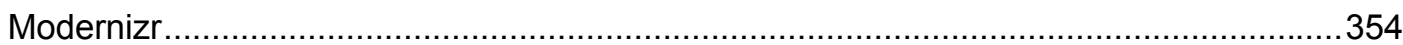

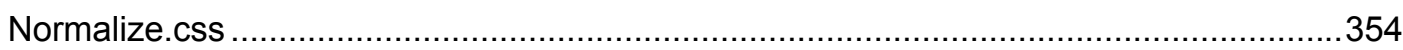

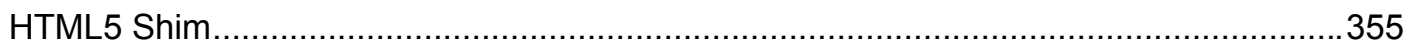

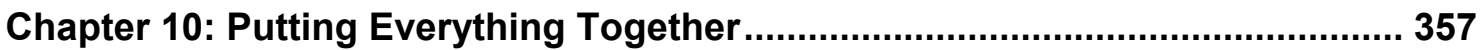

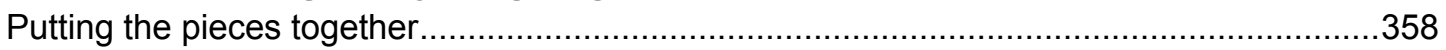

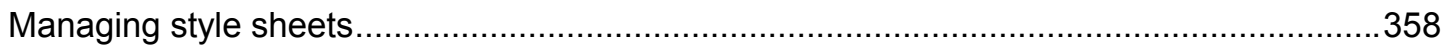

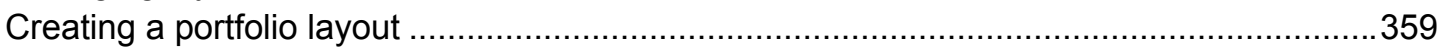

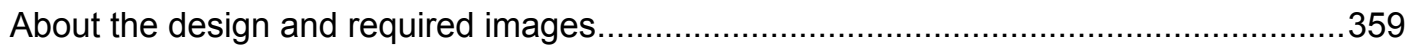

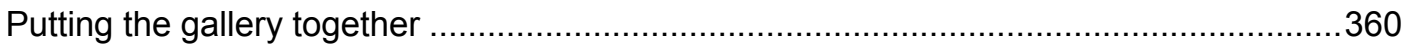

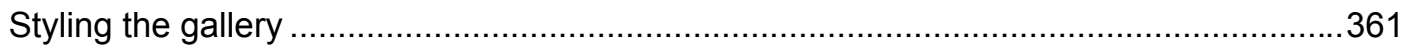

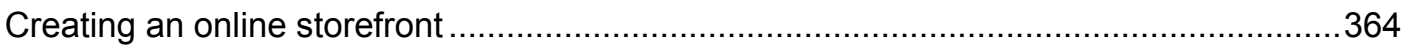

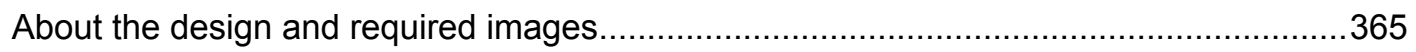

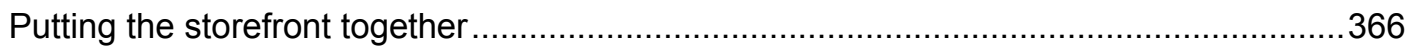

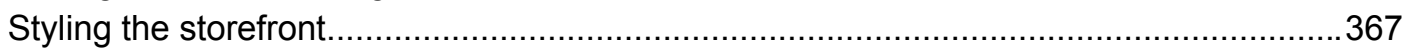

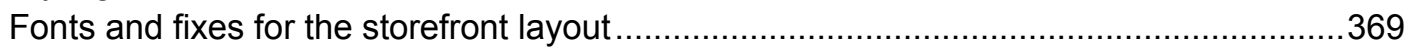

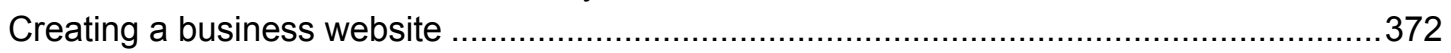

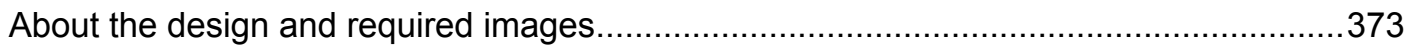

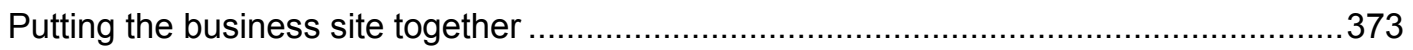

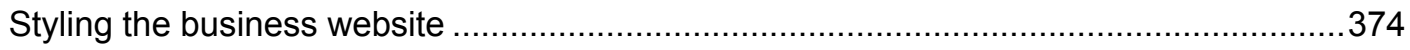

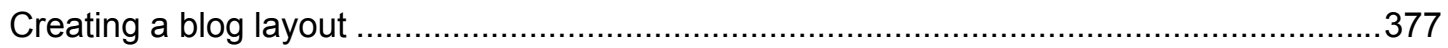

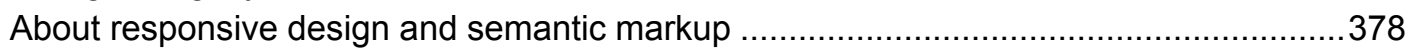

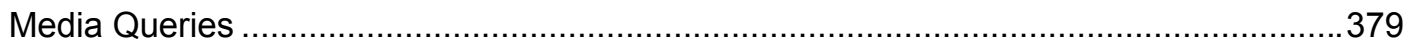

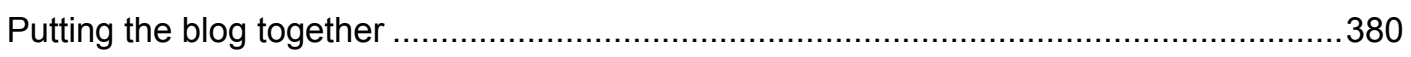

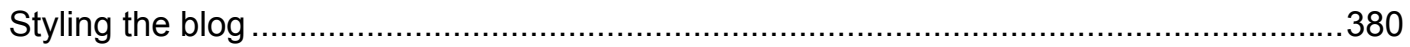

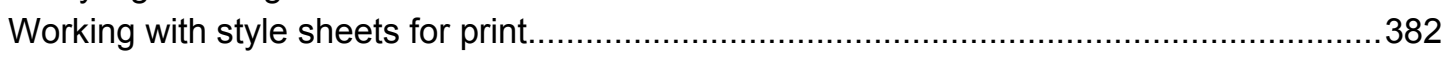

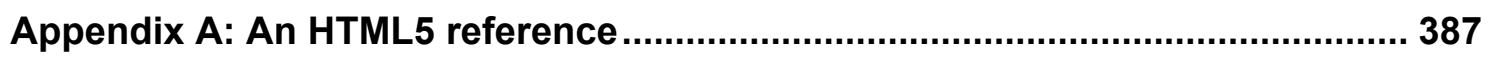

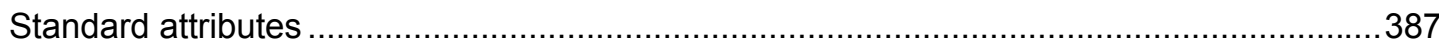

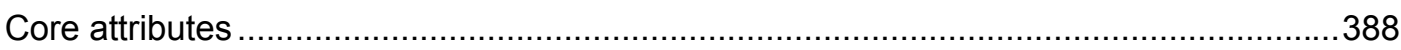

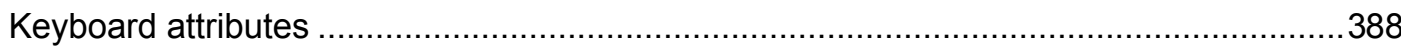

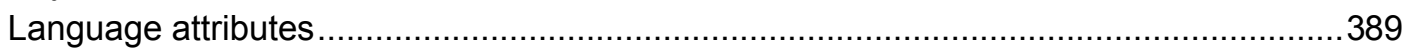

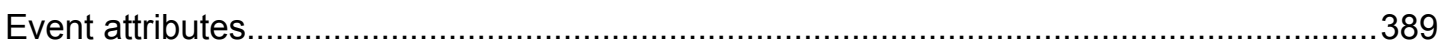

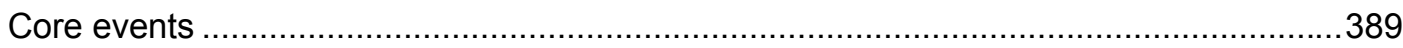

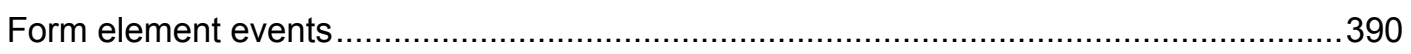


Window events 391

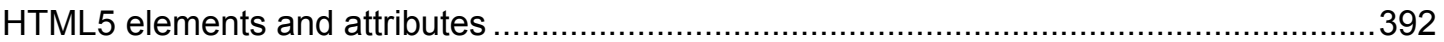

Appendix B: Web Color Reference ............................................................... 437

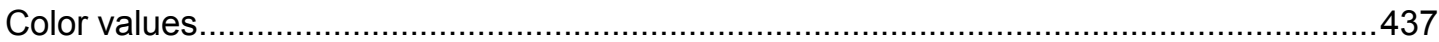

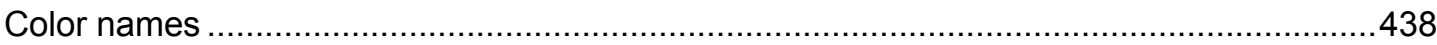

Appendix C: ENTITES reference ............................................................. 441

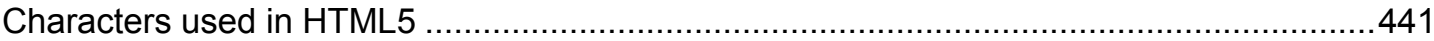

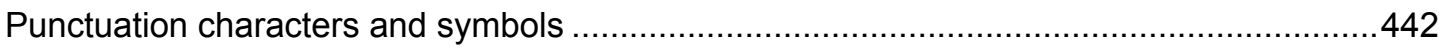

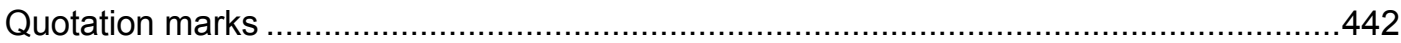

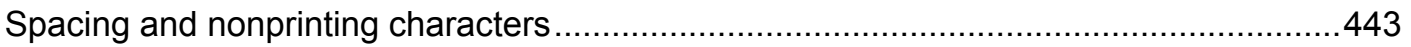

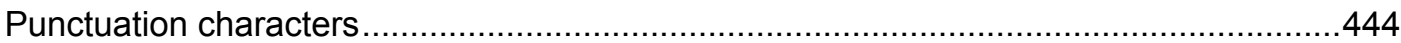

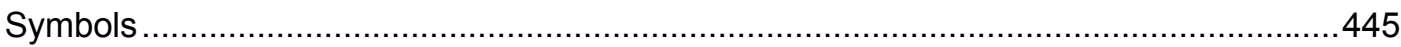

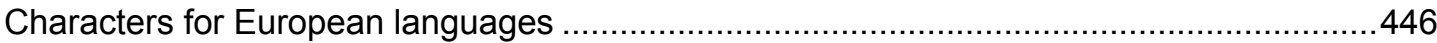

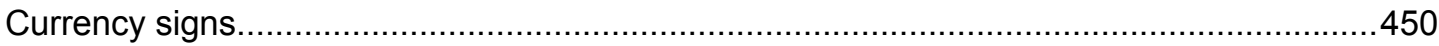

Mathematical, technical, and Greek characters ...................................................450

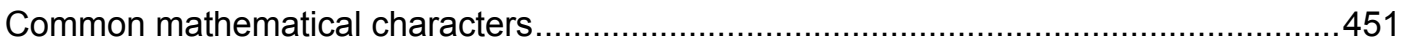

Advanced mathematical and technical characters ............................................. 451

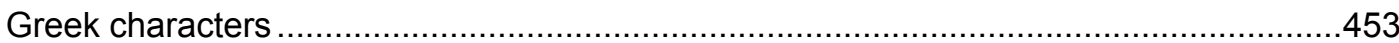

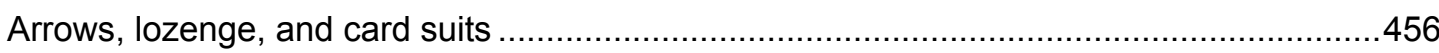

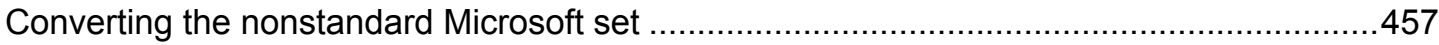

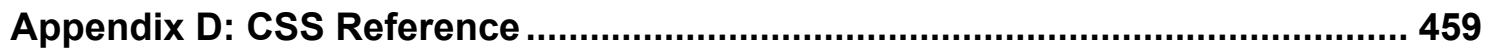

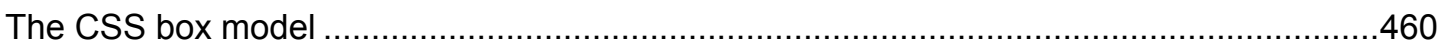

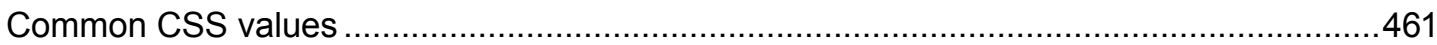

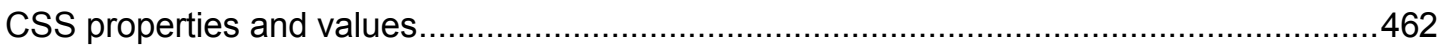

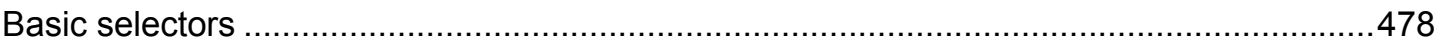

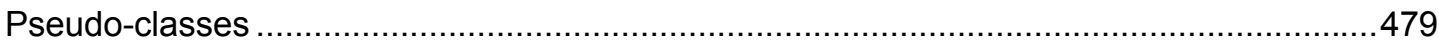

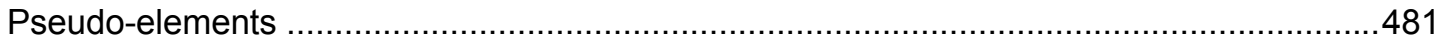

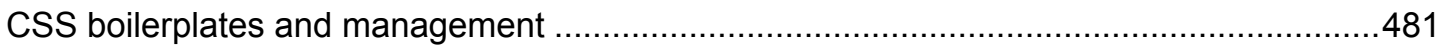

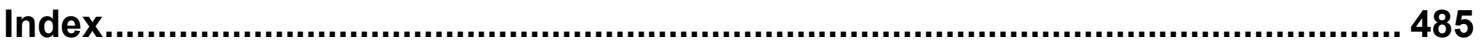




\section{About the Authors}

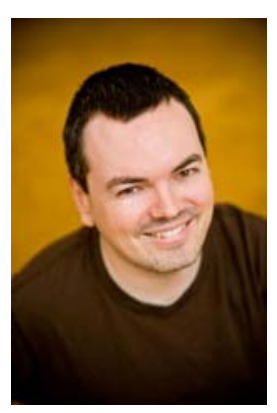

Craig Grannell is a writer and designer. Originally trained in the fine arts, the mid1990s saw Craig immersed in the world of digital media, his creative projects encompassing video, installation-based audio work, and strange live performancessometimes with the aid of a computer, televisions, videos, and a PA system, and sometimes with a small bag of water above his head. His creative, playful art, which contained a dark, satirical edge, struck a chord with those who saw it, leading to successful appearances at a number of leading European media arts festivals.

Craig soon realized he'd actually have to make a proper living, however. Luckily, the Web caught his attention, initially as a means to promote his art via an online portfolio but then as a creative medium in itself, and he's been working with it ever since. He founded tiny studio Snub Communications (www.snubcommunications.com) and has subsequently worked on design and writing projects for a diverse range of clients.

Along with writing the original version of the book you're holding right now (this version ably updated by Victor Sumner and Dionysios Synodinos), Craig has authored Web Designer's Reference (friends of ED, 2005) and various books on Dreamweaver. Elsewhere, he's penned numerous articles for Computer Arts, MacFormat, .net, Digital Arts, TechRadar, Tap!, and many other publications besides.

When not designing websites, Craig can usually be found hard at work in his quest for global superstardom by way of his eclectic audio project, the delights of which you can sample at www.projectnoise.co.uk.

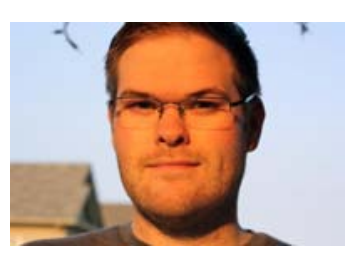

Victor Sumner is a senior software engineer at LookSmart, LTD, helping to build and maintain an online advertising platform. As a self-taught developer, he is always interested in emerging technologies and enjoys working on and solving problems that are outside his comfort zone.

When not at the office, Victor has a number of hobbies, including photography, horseback riding, and gaming. He lives in Ontario, Canada, with his wife, Alicia.

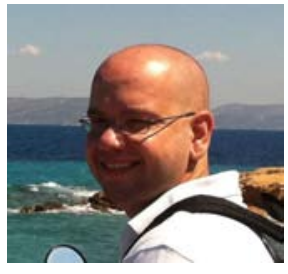

Dionysios Synodinos is the research platform team lead at C4Media and a freelance consultant, focusing on rich Internet applications, web application security, mobile web, and web services. He's the lead editor for HTML5 and JavaScript for InfoQ, where he regularly writes about the JVM platform. He's also the author of Pro HTML5 and CSS3 Design Patterns, published by Apress. Going back and forth between server-side programming and $\mathrm{UI}$ design for more than a decade, he has been involved in diverse software projects and has contributed to different technical publications. 


\section{About the Technical Reviewer}

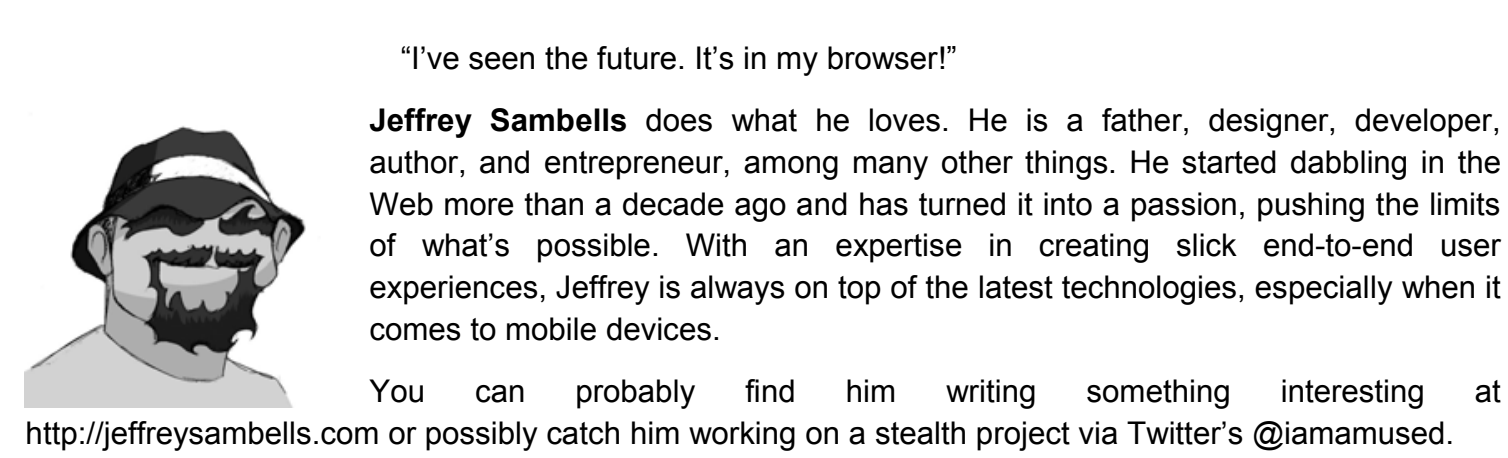




\section{About the Cover Image Artist}

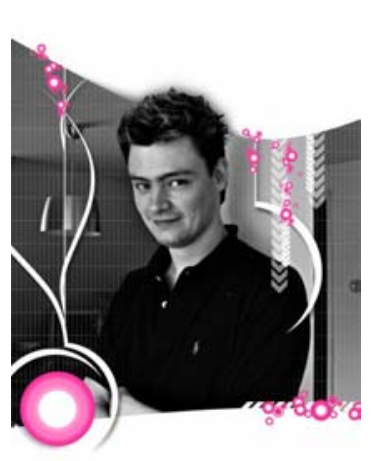

Corné van Dooren designed the front cover image for this book. After taking a break from friends of ED to create a new design for the Foundation series, he worked at combining technological and organic forms, with the results now appearing on the cover of this and other books.

Corné spent his childhood drawing on everything at hand and then began exploring the infinite world of multimedia-and his journey of discovery hasn't stopped since. His mantra has always been "the only limit to multimedia is the imagination," a saying that keeps him moving forward constantly.

Corné works for many international clients, writes features for multimedia magazines, reviews and tests software, authors multimedia studies, and works on many other friends of ED books. If you like Corné's work, be sure to check out his chapter in New Masters of Photoshop: Volume 2 (friends of ED, 2004). You can see more of his work (and contact him) at his website, www.cornevandooren.com. 


\section{Acknowledgments}

I would like to thank the Apress team for providing invaluable support putting this book together. Also, thanks to my wife and soul mate, Alicia, who continues to be supportive of everything I do and who inspires me to always do better.

-Victor Sumner

l'd like to thank Petros Efstathopoulos for motivating me to buy my first HTML book back in 1996 and for doing our first web programming together.

—Dionysios Synodinos 


\section{Introduction}

The Web is an ever-changing, evolving entity, and it's easy to get left behind. As designers and writers, we see a lot of books on web design, and although many are well written, few are truly integrated, modular resources that anyone can find useful in their day-to-day work. Most web design books concentrate on a single technology (or, commonly, a piece of software), leaving you to figure out how to put the pieces together.

\section{This book is different}

The Essential Guide to HTML5 and CSS3 Web Design provides a modern, integrated approach to web design. Each of the chapters looks at a specific aspect of creating a web page, such as formatting type, working with images, creating navigation, and creating layout blocks. In each case, relevant technologies are explored in context and at the appropriate times, just like in real-world projects; for example, markup is explored along with associated CSS and JavaScript, rather than each technology being placed in separate chapters, and visual design ideas are discussed so you can get a feel for how code affects page layouts. Dozens of practical examples are provided, which you can use to further your understanding of each subject. This highly modular and integrated approach means you can dip in and out of the book as you need, crafting along the way a number of web page elements that you can use on countless sites in the future.

Because the entire skills gamut is covered-from foundation to advanced-this book is ideal for beginners and longtime professionals alike. If you're making your first move into standards-based web design, the "ground floor" is covered, rather than an assumption being made regarding your knowledge. However, contemporary ideas, techniques, and thinking are explored throughout, ensuring that the book is just as essential for the experienced designer wanting to work on CSS layouts or for the graphic designer who wants to discover how to create cutting-edge websites.

This book's advocacy of web standards, usability, and accessibility with a strong eye toward visual design makes it of use to technologists and designers alike, enabling everyone to build better websites. For those moments when a particular tag or property value slips your mind, this book provides a comprehensive reference guide that includes important and relevant HTML5 elements and attributes, HTML5 entities, web colors, and CSS 3 properties and values.

\section{Code Examples}

Remember that you can also download files associated with this book from www.apress.com-just find the book and follow its instructions to access downloads and other associated resources.

To make it easier to work through the exercises, each one has an introductory box that lists where you can find any required files and the completed files within the downloadable file archive. A short overview of what you'll learn is also included. 